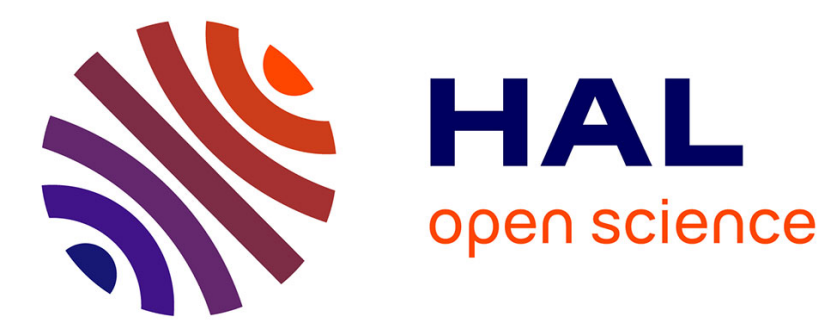

\title{
SOBRA - Shielding Optimization for BRAchytherapy
}

Guillaume Blin, Marie Gasparoux, Sebastian Ordyniak, Alexandru Popa

\section{To cite this version:}

Guillaume Blin, Marie Gasparoux, Sebastian Ordyniak, Alexandru Popa. SOBRA - Shielding Optimization for BRAchytherapy. 27th International Workshop on Combinatorial Algorithms, Aug 2016, Helsinki, Finland. hal-01331027

\section{HAL Id: hal-01331027 https://hal.science/hal-01331027}

Submitted on 20 Oct 2016

HAL is a multi-disciplinary open access archive for the deposit and dissemination of scientific research documents, whether they are published or not. The documents may come from teaching and research institutions in France or abroad, or from public or private research centers.
L'archive ouverte pluridisciplinaire HAL, est destinée au dépôt et à la diffusion de documents scientifiques de niveau recherche, publiés ou non, émanant des établissements d'enseignement et de recherche français ou étrangers, des laboratoires publics ou privés. 


\title{
SOBRA - Shielding Optimization for BRAchytherapy ${ }^{\star}$
}

\author{
Guillaume Blin ${ }^{1}$, Marie Gasparoux ${ }^{1}$, Sebastian Ordyniak ${ }^{2}$, and Alex Popa ${ }^{3}$ \\ 1 Univ. Bordeaux, LaBRI, CNRS UMR 5800, F-33400 Talence, France \\ ${ }^{2}$ Institute of Information Systems, TU Wien, Vienna, Austria \\ 3
}

\begin{abstract}
In this paper, we study a combinatorial problem which arises in the development of innovative treatment strategies and equipment using tunable shields in internal radiotherapy. From an algorithmic point of view, the problem is related to circular integer word decomposition into circular binary words under constraints. We consider several variants of the problem, depending on constraints and parameters and present polynomial time exact algorithms, polynomial time approximation algorithms and NP-hardness results.
\end{abstract}

\section{Introduction}

In France, every year, almost 200.000 patients are treated by radiotherapy as part of their cancer treatment. This kind of therapy is using ionizing radiation aiming at controlling or killing malignant cells as a curative procedure or as part of adjuvant therapy and is widely used (in $2 / 3$ of the cancer treatments). While internal radiotherapy treatments are currently widespread and considered as routine, there is still room for related innovative developments. The aim is to concentrate the radiation beams as precisely as possible towards the tumor site while sparing as much as possible normal tissues such as skin or organs which radiation must pass through to treat the tumor (the so-called organs at risk).

Brachytherapy - also sometimes referred to as Curietherapy - refers to a short distance (brachys in Greek) treatment of cancer with radiation from small, encapsulated radionuclide sources. Brachytherapy uses encapsulated radioactive sources (also called seeds) to deliver a high dose to tissues near the source. It is characterized by strong dose gradients, i.e., the dose becomes negligible in a very short distance from the source (about $10 \%$ per $\mathrm{mm}$ ) [6]. This type of treatment is given by placing sources directly into or near the volume to be treated. The dose is then delivered continuously, either over a short period of time (temporary implants) or over the lifetime of the source to a complete decay (permanent implants). There are many different techniques and sources available.

\footnotetext{
* This research was partially supported by PoPRA project funded by Conseil Régional d'Aquitaine and European FEDER and IdEx Bordeaux. We thank Maxence Ronzié for initial assistance in the very first step of the project.
} 
In this contribution, we focus on High Dose Rate implants. High dose rate or HDR brachytherapy is a form of internal radiation which temporarily expose abnormal tissue to a high amount of radiation. Under Computed Tomography (CT) and Fluoroscopy guidance, a bronchoscope or a needle is used to deliver a catheter into a position at the tumor site. The other end of this catheter is connected to a computerized machine. This machine passes a small radioactive metal seed through the catheter. The catheter guides the seed to the tumor site. The seed moves step by step through the catheter in order to cover the whole tumor site. The time spent at each position - also known as dwell time - is used to control the radiation dose in different regions of the tumor. The overall effect of HDR brachytherapy is to deliver short and precise amounts of high-dose radiation to a tumor while minimizing healthy tissue exposure. After a series of treatments the catheter is removed leaving no radioactive seeds in the body.

One of the main drawback of this technique comes from the lack of precise modulation of the irradiation field and thus the conformation to the shape of the tumor site. In this paper, we aim at studying the benefit of an innovative modulation technique in brachytherapy using tunable shields (as done in external radiotherapy). This approach will allow accumulating both the temporal modulation currently used and the shielding modulation. The aim is to provide treatment of better accuracy by adapting more precisely to the tumor shape. Indeed, currently, the modulation of the radiation source is done by controlling the time spent at each position by the source along the catheter. The main problem is that, at any position, the irradiation is uniform and can be represented as a cylinder surrounding the catheter. This shape does not always conform to the relative placement of the tumor and the organs at risks (i.e., in the radiation field). In this contribution, we consider the technic which aim at modulating a unique radioactive source using a gear inspired by external radiotherapy.

The use of the shield will allow to preserve, for a given position along the catheter, some part of the surrounding area. The so-called rotating shield brachytherapy (RSBT) was conceptually proposed by Ebert in 2002 [2]. In RSBT the dose is delivered through a partially shielded radiation source in an optimized step-shot fashion (as done in classical brachytherapy treatment) to improve tumor dose conformity. The intensity of radiation is modulated by the amount of time the shield is pointed in a given direction. RSBT $[5,4,12]$ and other intensitymodulated brachytherapy techniques such as dynamic modulated brachytherapy (DMBT) $[11,10,9]$ were further studied with the aim of improving intracavitary brachytherapy dose distributions for rectal and cervical cancer. We will first focus on a peculiar type of shield which have been briefly described in the patent [8] and studied in [3]. It corresponds to a set of shield segments forming a cylinder that can be individually retracted to produce circumferentially limited radiation output, directed radially. According to the way the sources are introduced in the patient body, and the physical constraints of the material, it is not possible to build sector of size as small, and thus as high resolution, as wanted. Therefore, using the possible rotation of the equipment, the aim is to find a sequence of sectors configurations that allows delivering a dose as near as possible to the 
prescribed dose. The corresponding algorithmic aspects are unexplored and the goal of this paper is to conduct an algorithmic study which will guide the final development of the equipment. From an algorithmic point of view, the problem is related to circular integer word decomposition into circular binary words under constraints that we formaly introduced in Section 2.

\section{Preliminaries}

Considering each dwell position of the irradiation source (denoted $I$ ), our main objective is to deliver to each part of the surrounding volume its proper irradiation dose. For this purpose, we will use a paddle-based shielding equipment $P$ of $K$ paddles (also referred as sectors for ease) that can stop the radiation going through when they are not retracted. We will consider the surrounding volume to be treated as a circular volume of interest divided in $N$ subvolumes. In the following, a treatment plan for a given dwell position will be defined as a sequence of $T$ shield configurations $\left(\left(P^{1}, \tau^{1}\right),\left(P^{2}, \tau^{2}\right) \ldots\left(P^{T}, \tau^{T}\right)\right)$ where $P^{t}, 1 \leq t \leq T$, is a paddle configuration and $\tau^{t}$ is its dwell time. Each paddle configuration is represented as a binary string $P^{t}=p_{0}^{t} p_{1}^{t} \ldots p_{K-1}^{t}$ where $p_{k}^{t}$ represents the state (open or closed) of the sector $k$ of $P^{t}$. An open sector of the shield (paddle retracted allowing radiation going through) is represented by a 1 , while a closed one (paddle is out and radiation is stopped) is represented by a 0 .

For each given step $\left(P^{t}, \tau^{t}\right)$ in the treatment plan, a corresponding received doses $D^{t}$ by the surrounding volume is defined as a string of integers $D^{t}=$ $d_{0}^{t} d_{1}^{t} \ldots d_{N-1}^{t}$ where $d_{n}^{t}$ corresponds to the total irradiation time the subvolume $n$ was exposed to during this step. Roughly, it corresponds to the contribution of the corresponding treatment step to the whole treatment plan. For ease, without loss of generality, when parameters $P^{t}$ and $\tau^{t}$ are not informative, we will write $D$. Regarding the entire treatment plan, we will denote the prescribed doses as a string of nonnegative integers $\hat{D}=\hat{d}_{0} \hat{d}_{1} \ldots \hat{d}_{N-1}$ where $\hat{d}_{n}$ corresponds to the total irradiation time needed to achieve the right dose for the subvolume $n$. We will moreover denote the total received doses as a string of integers $D=d_{0} d_{1} \ldots d_{N-1}$, such that for all $d_{n} \in D, d_{n}=\sum_{1<t<T} d_{n}^{t}$.

For ease and without loss of generality, we assume that each shield sector is associated to $w=N / K$ consecutive patient volumes, and, for simplicity, that $K$ divides $N$ (so $w$ is an integer). By default, each shield sector $p_{k}$ will be associated to $D_{k}=D[k \cdot w,(k+1) \cdot w-1]=d_{k \cdot w} d_{k \cdot w+1} \ldots d_{k \cdot w+w-1}$ of length $w$ (see example Figure 1a). We can remark that $D=D_{0} D_{1} \ldots D_{K}$. Informally, one may see $P$ and $D$ as circular strings, $P$ placed inside $D$ and representing a mask that can stop the radiation from going through (see Figure 1b, with a counterclockwise indexation). In future work, planned for the full version of this paper, we plan to explore a variant of the problem where one will be able to change this association by applying a rotation of the shield represented by $P$.

Let us consider the practical case where one is applying a given shielded configuration (represented by $P$ ) on a patient (represented by $D$ ) for a given amount of time $\tau$ (expressed in a given unit of time). Let us denote $D(P, \tau)=$ 


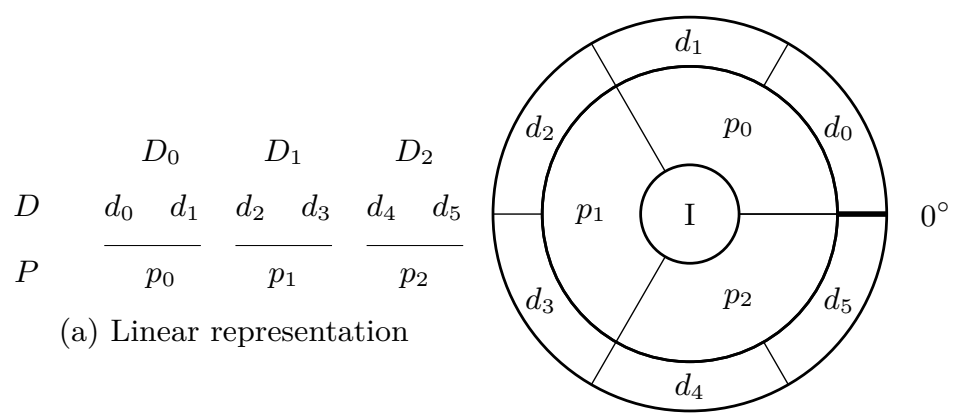

(b) Circular representation

Figure 1: Relation between $P$ and $D(K=3, N=6)$

$d_{0} d_{1} \ldots d_{N-1}$ the string of integers obtained by applying radiation for a time $\tau$ to $D$ through the mask $P$. We consider that $p_{k}=1$ (resp. $p_{k}=0$ ) denotes applying radiation (resp. no radiation applied) to the area $D_{k}$. Moreover, $d_{n}=\tau$ (resp. $d_{n}=0$ ) if radiation is applied to the volume $n$ for a time $\tau$ (resp. if no radiation is applied there). In other words, each patient volume associated to an open sector (represented by a 1 ) is irradiated $\tau$ units of time, while volume associated to a closed sector (represented by a 0 ) is left in its previous state.

One may consider several variants of the problem, depending on constraints and parameters. First of all, the shield configuration can be considered as fixed or dynamic (one fixed mask or a minimal number of chosen masks) and provided with or without rotation capabilities (this last property is not considered here). This properties are related to manufacturing purposes and constraints. We moreover consider allowing or not irradiation overdoses $\left(d_{n}>\hat{d}_{n}\right)$. Indeed, in practice, it is convenient to overdose a tumor region while one should try to not overdose organ at risks regions. From a combinatorial point of view, there are two parameters that alter the overall treatment time; namely, the sum of the irradiation times and the number of configurations (as a transition between two configurations will require some time). In the following, we will consider variants of the problem based on the previous observations. In the first two variants, the input consists of only one shield configuration that is given and fixed. The goal is to decide what is the optimum amount of radiation that can be applied when allowing or not overdoses. As proven in Section 3, these variants of the problem are polynomial time solvable.

Problem 1 (FIXMASK). Given a prescribed dose represented as a string of nonnegative integers $\hat{D}=\hat{d}_{0} \hat{d}_{1} \ldots \hat{d}_{N-1}$ and a fixed shield configuration represented as a binary string $P=p_{0} p_{1} \ldots p_{K-1}$, find the dwell time $\tau$ minimizing $\sum_{n=0}^{N-1}\left|\hat{d}_{n}-d_{n}\right|$ with $D=D(P, \tau)$ s.t. $\forall n<N$.

While in FIXMASK variant of the problem, $\hat{d}_{n}-d_{n}$ can be negative - that is overdoses are allowed - in FIXMASK ${ }^{+}$variant, we moreover impose that $\forall n<N$, 
$d_{n} \leq \hat{d}_{n}$ - thus, forbidding overdoses. We now consider variants of the problem where multiple shield configurations are allowed. As mentioned previously, two different criteria can be optimized in such a treatment plan. One would like to either achieve the optimal difference between the prescribed dose and the actual total delivered dose using a minimal number of shield configurations or given an upper bound on the number of shield configurations, achieving the minimum reachable difference. From a computational point of view, there is no evidence that these problems are equivalent. Formally, the problems are defined as follows.

Problem 2 (MinFIXMASKS ${ }_{\mathrm{OPT}}$ ). Given two nonnegative integers $K$ and diff and a string of integers $\hat{D}=\hat{d}_{0} \hat{d}_{1} \ldots \hat{d}_{N-1}$ (with $N$ being a multiple of $K$ ), find a treatment plan $\left(\left(P^{1}, \tau^{1}\right),\left(P^{2}, \tau^{2}\right) \ldots\left(P^{T}, \tau^{T}\right)\right)$ minimizing $T$ such that $\sum_{n=0}^{N-1}\left|\hat{d}_{n}-d_{n}\right|<$ diff, where $\forall d_{n} \in D, d_{n}=\sum_{1 \leq t \leq T} d_{n}^{t}$.

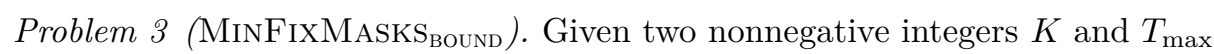
and a string of integers $\hat{D}=\hat{d}_{0} \hat{d}_{1} \ldots \hat{d}_{N-1}$ (with $N$ being a multiple of $K$ ), find a treatment plan $\left(\left(P^{1}, \tau^{1}\right),\left(P^{2}, \tau^{2}\right) \ldots\left(P^{T}, \tau^{T}\right)\right)$ where $T<T_{\max }$ minimizing $\sum_{n=0}^{N-1}\left|\hat{d}_{n}-d_{n}\right|$, where $\forall d_{n} \in D, d_{n}=\sum_{1 \leq t \leq T} d_{n}^{t}$.

Similarly to in FixMASK ${ }^{+}$, in MinFixMASKS OPT $^{+}$and MinFIXMASKS BOUND $^{+}$ variants of the problem, we moreover impose that $\forall n<N, d_{n} \leq \hat{d}_{n}-$ thus, forbidding overdoses.

\section{Polynomial Results}

In this section, we show that the variants of the problem where the shield configuration is given and fixed are solvable in polynomial time. Clearly, for a fixed masked, the doses associated to closed paddles cannot be brought closer to the corresponding prescribed doses and will thus not be considered.

Theorem 1. FIXMASK ${ }^{+}$can be solved in $\mathcal{O}(N)$.

Proof. Obviously, the maximal irradiation time $\tau_{\max }$ that can be applied is the minimum $\hat{d}_{j}$ of $\hat{D}$ associated to a 1 bit of $P$, since any greater value would decrease $\hat{d}_{j}-d_{j}$ below 0 . Therefore, $\tau_{\max }=\min \left\{\hat{d}_{j} \in \hat{D}: p_{\lfloor j / N\rfloor}=1\right\}$ which leads to an overall difference of $\sum_{n=0}^{N-1}\left(\hat{d}_{n}-d_{n}\right)=\sum_{n=0}^{N-1} \hat{d}_{n}-\sum_{p_{k} \in P}\left(p_{k} \cdot w \cdot \tau_{\max }\right)$ (where $w=N / K) . \tau_{\max }$ can be obtained by browsing $\hat{D}$ in linear complexity.

The main observation required to show that FIXMASK can also be solved in polynomial time is given in the following lemma.

Lemma 1. For a sequence $S$ of natural numbers and a natural number $x$ consider the function $f(x)$ with $f(x)=\sum_{s \in S}|s-x|$. Then $f(x)$ has a unique minimum, which is only reached by any number $x$ in between the at most two medians of $S$. Moreover, for any $x$ not between the at most two medians of $S$, the function $f(x)$ decreases with the distance of $x$ to a median of $S$. 
The above lemma implies that an optimum dwell time for an instance of FIXMASK is a median of the subsequence of $\hat{D}$ containing all prescribed doses for which the paddles are open.

Theorem 2. FIXMASK can be solved in $\mathcal{O}(N \log N)$.

Proof. Because of Lemma 1 the best possible value that we can achieve for $\sum_{n=0}^{N-1}\left|\hat{d}_{n}-d_{n}\right|$ is obtained by setting the dwell time $\tau_{0}$ to any median of the subsequence of $\hat{D}$ containing only the prescriped doses for which the paddles are open (in the given mask). A median of this sequence can be found by sorting the corresponding set in time $\mathcal{O}(N \log N)$.

\section{Quasi-polynomial Algorithms for MinFixMasks}

In this section, we present exact algorithms for all variants of the MiNFIXMASKS problem. The presented algorithms run in quasi-polynomial time if the values of the precribed patient doses are bounded by a polynomial in the number of precribed doses. As a by product we show that the problems MiNFIXMASKS ${ }_{\text {OPT }}$ and MINFIXMASKS ${ }_{\mathrm{OPT}}^{+}$can be approximated in polynomial-time within a factor of $\log \hat{d}_{\max }$ of the optimum where $\hat{d}_{\max }$ is the maximum prescribed dose to a subvolume of the patient, i.e., $\hat{d}_{\max }:=\max _{\hat{d}_{n} \in \hat{D}} \hat{d}_{n}$. We first show that it is sufficient to consider treatment plans where the applied dwell times are pairwise distinct.

Lemma 2. For any instance of MinFIXMASKS ${ }_{\mathrm{OPT}}$, MinFIXMASKS OPT $^{+}$, MINFIXMASKS $_{\mathrm{BOUND}}$, and MINFIXMASKS $\mathrm{BOUND}^{+}$there is an optimal solution $\left(\left(P^{1}, \tau^{1}\right),\left(P^{2}, \tau^{2}\right) \ldots\left(P^{T}, \tau^{T}\right)\right)$ satisfying $\tau^{i} \neq \tau^{j}$ for every $i$ and $j$ with $1 \leq i \neq j \leq T$.

Proof. Let $\mathcal{P}=\left(\left(P^{1}, \tau^{1}\right), \ldots\left(P^{T}, \tau^{T}\right)\right)$ be an optimal solution of an instance $\mathcal{I}$ of any of the variants of MinFixMAsks problem. Let $i$ and $j$ with $1 \leq i \neq j \leq T$ be such that $\tau^{i}=\tau^{j}$. Let (i) $\tau_{*}^{j}=2 \tau^{j}$, (ii) the binary string $P_{*}^{i}$ be obtained by the $X O R$ of binary strings $P^{i}$ and $P^{j}$, and (iii) the binary string $P_{*}^{j}$ be obtained by the $A N D$ of binary strings $P^{i}$ and $P^{j}$. Then the treatment plan obtained from $\mathcal{P}$ by replacing $\left(P^{i}, \tau^{i}\right)$ with $\left(P_{*}^{i}, \tau_{*}^{i}\right)$ and $\left(P^{j}, \tau^{j}\right)$ with $\left(P_{*}^{j}, \tau_{*}^{j}\right)$ is also an optimal solution of $\mathcal{I}$. Moreover, by applying this procedure iteratively we eventually obtain an optimal solution of $\mathcal{I}$ such that all dwell times are pairwise distinct.

Let $S$ be a set of dwell times. We say that $S$ is complete if it contains a subset $S^{\prime}$ for every number $1 \leq i \leq \hat{d}_{\max }$ such that $i=\sum_{s \in S^{\prime}} s$. We say that a treatment plan is $S$-restricted if it uses only dwell times from $S$ and each of them at most once.

Lemma 3. Let $S$ be a set of dwell times. Then an $S$-restricted treatment plan minimizing $\sum_{n=0}^{N-1}\left|\hat{d}_{n}-d_{n}\right|$ can be found in time $O\left(\left(\hat{d}_{\max }\right)^{2}|S|+w^{2} \log w+\right.$ $\left.K \hat{d}_{\max }\right)$. Moreover, an S-restricted treatment plan minimizing $\sum_{n=0}^{N-1}\left|\hat{d}_{n}-d_{n}\right|$ 
under the additional constraint $\hat{d}_{n}-d_{n} \geq 0$ for every $0 \leq n \leq N-1$ can be found in $O\left(\left(\hat{d}_{\max }\right)^{2}|S|+K w+K \hat{d}_{\max }\right)$ time. Moreover, if $\bar{S}$ is complete then the $S$-restricted treatment plans returned by the above algorithms are optimal among all (not necessarily $S$-restricted) treatment plans.

Lemma 4. There is a treatment plan minimizing $\sum_{n=0}^{N-1}\left|\hat{d}_{n}-d_{n}\right|$ using at most $\left\lfloor\log \hat{d}_{\text {max }}\right\rfloor+1$ steps. Moreover, such a treatment plan can be found in polynomial time. The same holds for a treatment plan minimizing $\sum_{n=0}^{N-1}\left|\hat{d}_{n}-d_{n}\right|$ under the additional constraint that $\hat{d}_{n}-d_{n} \geq 0$ for every $n$ with $0 \leq n \leq N-1$.

Proof. Because the set $S=\left\{2^{i}: 0 \leq i \leq\left\lfloor\log \hat{d}_{\text {max }}\right\rfloor\right\}$ is complete and has size $\left\lfloor\log \hat{d}_{\text {max }}\right\rfloor+1$, this follows immediately from Lemma 3 .

Because any non-trivial instance of MinFixMASKS ${ }_{\mathrm{OPT}}$ and MinFixMASKS ${ }_{\mathrm{OPT}}^{+}$ require at least one step, we obtain the following corollary from the above lemma.

Corollary 1. MINFIXMASKS ${ }_{\mathrm{OPT}}$ and MINFIXMASKS $\mathrm{OPT}_{\mathrm{OPT}}^{+}$can be approximated in polynomial time within a factor of $\log \hat{d}_{\max }$ of the optimum.

We are now ready to show our main theorem of this section.

Theorem 3. MINFIXMASKS ${ }_{\mathrm{OPT}}$ and MINFIXMASKS BOUnd $_{\text {can }}$ be solved in time $\left.O\left(\hat{d}_{\max }^{\left.\log \hat{d}_{\max }\right\rfloor+1}\left(\hat{d}_{\max }\right)^{2}\left(\left\lfloor\log \hat{d}_{\max }\right\rfloor+1\right)+w^{2} \log w+K \hat{d}_{\text {max }}\right)\right)$. Moreover, MINFIXMASKS ${ }_{\mathrm{OPT}}^{+}$and MINFIXMASKS ${ }_{\mathrm{BOUND}}^{+}$can be solved in time $\left.O\left(\hat{d}_{\max }^{\left\lfloor\log \hat{d}_{\text {max }}\right\rfloor+1}\left(\hat{d}_{\max }\right)^{2}\left(\left\lfloor\log \hat{d}_{\max }\right\rfloor+1\right)+K w+K \hat{d}_{\max }\right)\right)$.

Proof. The algorithm goes over all sets $S$ containing at most $\left\lfloor\log \hat{d}_{\text {max }}\right\rfloor+1$ (respectively at most $\min \left\{\left\lfloor\log \hat{d}_{\text {max }}\right\rfloor+1, T_{\max }\right.$ in the case of MinFIXMASKS Bound and MinFIXMASKS ${ }_{\text {Bound }}^{+}$) dwell times between 1 and $\hat{d}_{\text {max }}$. For every such set $S$, the algorithm then uses Lemma 3 to compute the optimal (the meaning of optimal here depends on the considered problem) $S$-restricted treatment plan. Finally, the algorithm outputs the best treatment plan that satisfies $\sum_{n=0}^{N-1}\left|\hat{d}_{n}-d_{n}\right|<$ diff found for any considered set $S$, or the algorithm returns failure if no treatment plan exists with $\sum_{n=0}^{N-1}\left|\hat{d}_{n}-d_{n}\right|<d i f f$. The stated running of the algorithm follows because there are at most $\hat{d}_{\max }^{\left.\log \hat{d}_{\max }\right\rfloor+1}$ such sets $S$ and for each of them, we require time at most $\left.O\left(\hat{d}_{\max }\right)^{2}|S|+w^{2} \log w+K \hat{d}_{\max }\right)$ respectively time at most $O\left(\left(\hat{d}_{\max }\right)^{2}|S|+K w+K \hat{d}_{\text {max }}\right.$ ) (in the case of the problems MinFIXMASKS OPT $_{\text {Th }}^{+}$and MINFIXMASKS $\left._{\text {BOUND }}^{+}\right)$. The correctness of the algorithm follows from Lemmas 2, 3 and 4.

Corollary 2. MINFIXMASKS ${ }_{\mathrm{OPT}}^{+}$can be solved in quasi-polynomial time if $\hat{d}_{\text {max }}$ is bounded by a polynomial in the size of the input instance. 


\section{$5 \quad$ Hardness of MinFixMasks opt $_{\text {and MinFixMasks }}$ aound}

In this section, we provide the first hardness results on the MiNFIXMASKS ${ }_{\mathrm{OPT}}$

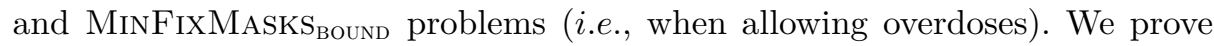
our hardness results via a reduction from the Monotone 1-3 SAT problem (proven to be NP-complete in [7]): Given a boolean formula $\phi=\left\{c_{1}, c_{2}, \ldots\right\}$ in 3-CNF of $|\phi|$ clauses built on a set $V=\left\{v_{1}, v_{2}, \ldots\right\}$ of $|V|$ variables, such that its clauses contain only unnegated literals, does there exist a truth assignment on $V$ satisfying $\phi$ such that each clause is satisfied by exactly one of its three literals?

We first prove that both the MINFIXMASKS ${ }_{\mathrm{OPt}}$ and MinFIXMASKS BOUnd problems are NP-hard even when each shield sector is associated to two consecutive patient volumes (i.e., $w=2)$. Given any instance $(\phi, V)$ of Monotone 1-3 SAT problem, we build an instance of MinFixMASKS as follows. For all $i \in[1,|V|]$, let $q_{i}$ be an integer value computed using the following recurrence formula: $q_{i}=1+2 \times \sum_{j=1}^{i-1}\left(1+q_{j}\right)$ with $q_{1}=|V|$. For each variable $v_{i} \in V$, we build the sequence $V_{i}=\left(q_{i}, 1+q_{i}\right)$. For each clause $c_{m}=\left(v_{a}, v_{b}, v_{c}\right) \in \phi$, we build the sequence $C_{m}$ composed of two copies of $\left(q_{a}+q_{b}+q_{c}+2\right)$. For each pair $\left(v_{i}, v_{j}\right), i<j \leq|V|$, we build the sequence $V_{i, j}=\left(q_{i}+q_{j}, q_{i}+q_{j}+2\right)$. Let $V_{*, j}$ be the concatenation of $V_{1, j}, V_{2, j}, \ldots V_{j-1, j}$. The sequence $\hat{D}$ is obtained by concatenating in order $V_{1} V_{2} \ldots V_{|V|} C_{1} C_{2} \ldots C_{|\phi|} V_{*, 2} V_{*, 3} \ldots V_{*,|V|}$. We finally set $K=|V|+|\phi|+\frac{|V| \cdot(|V|-1)}{2}, N=2 \cdot K(i . e ., w=2)$. For the MinFixMASKS ${ }_{\text {Bound }}$ variant, we further set $T_{\max }=|V|$. An illustration is given in Figure 2 .

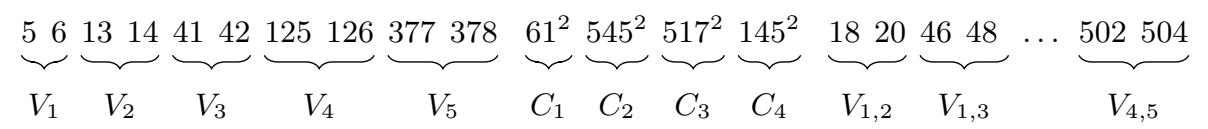

Figure 2: Example of an instance of MinFIXMASKS considering the boolean formula $\phi=\left(v_{1}, v_{2}, v_{3}\right) \wedge\left(v_{3}, v_{4}, v_{5}\right) \wedge\left(v_{2}, v_{4}, v_{5}\right) \wedge\left(v_{1}, v_{2}, v_{4}\right)$ which only admits one optimal solution $\left(v_{1}=v_{5}=\right.$ true and $v_{2}=v_{3}=v_{4}=$ false) where $N=18$ and $m=5$. For ease of notation, $v^{x}$ will denote $x$ occurrences of the element $v$ (thus $61^{2}$ corresponds to 6161 ) and most elements $V_{i, j}$ have been ommited.

One can first state some interesting properties of this construction (the proof is omitted here and provided in Appendix).

Lemma 5. To achieve a solution with diff $=|V|^{2}$ (which is the optimal solution one can get), one has to use at least $|V|$ different configurations.

Proposition 1. The MINFIXMASKS ${ }_{\mathrm{OPT}}$ and MINFIXMASKS $\mathrm{BOUND}_{\text {M }}$ problems are NP-hard when $w=2$.

Proof. Let us prove that, if $w=2$, the MinFixMASKS $\operatorname{OPT}_{\mathrm{O}}$ and MinFixMASKS $_{\text {Bound }}$ problems are equivalent to the Monotone 1-3 SAT problem. 
$(\Leftarrow)$ Given any truth assignment satisfying $\phi$ such that each clause is satisfied by exactly one of its literals, let us build a treatment plan consisting of a set of $|V|$ binary strings $\left\{P^{1}, P^{2}, \ldots P^{|V|}\right\}$ with the corresponding irradiation times $\left\{\tau^{1}, \tau^{2}, \ldots \tau^{|V|}\right\}$ as follows. We build a a set of $|V|$ binary strings $\left\{P^{1}, P^{2}, \ldots P^{|V|}\right\}$ with the corresponding irradiation times $\left\{\tau^{1}, \tau^{2}, \ldots \tau^{|V|}\right\}$, using a given truth assignment over $\phi$.

For all $1 \leq n|V|, \tau^{n}$ is defined accordingly to the boolean value $v_{n}$ as $\tau^{n}=q_{n}$ if $v_{n}=$ true; $\tau^{n}=1+q_{n}$ otherwise. Each $P^{n}$ is obtained by concatenating three substrings corresponding to the $V_{i}$ 's, $C_{m}$ 's and $V_{i, j}$ 's : $P^{n}=P_{\mathrm{V}}^{n} P_{\mathrm{C}}^{n} P_{\mathrm{V}_{*}}^{n}$ where $P_{\mathrm{V}}^{n}=0^{n-1} 10^{|V|-n}, P_{\mathrm{C}}^{n}=\operatorname{In}(n, 1) \operatorname{In}(n, 2) \ldots \operatorname{In}(n,|\phi|)\left(\operatorname{In}(n, m)\right.$ is 1 if $v_{n} \in c_{m}$, 0 otherwise) and $P_{\mathrm{V}_{*}}^{n}=P_{\mathrm{V}_{*, 1}}^{n} P_{\mathrm{V}_{*, 2}}^{n} \ldots P_{\mathrm{V}_{*,|V|}}^{n}$. Each $P_{V_{*}, i}^{n}$ is defined accordingly to $i$ and $n$ as $P_{V_{*, i}}^{n}=0^{i-1}$ if $i<n ; P_{V_{*, i}}^{n}=1^{n-1}$ if $i=n$ and $P_{V_{*, i}}^{n}=0^{n-1} 10^{i-1-n}$ otherwise.

By construction, with the obtained treatment plan, a total dwell time $\tau^{n}$ (either $q_{n}$ or $1+q_{n}$ ) is applied to each $V_{n}$. Moreover, any $C_{m}$ corresponding to a clause $\left(v_{a}, v_{b}, v_{c}\right)$ receives a total dwell time of $q_{a}+q_{b}+q_{c}+2$, since by hypothesis exactly one of $\left\{v_{a}, v_{b}, v_{c}\right\}$ is true in our assignment: that is either $q_{a}+\left(1+q_{b}\right)+\left(1+q_{c}\right)$ or $\left(1+q_{a}\right)+q_{b}+\left(1+q_{c}\right)$ or $\left(1+q_{a}\right)+\left(1+q_{b}\right)+q_{c}$. Finally, a total dwell time $\tau^{i}+\tau^{j}$ such that $q_{i}+q_{j} \leq \tau^{i}+\tau^{j} \leq q_{i}+q_{j}+2$ has been applied to each $V_{i, j}$, lowering its cost to 2. Thus, any Monotone 1-3 SAT solution over $\phi$ give us an optimal solution for our instance of MiNFIXMASKS using $|V|$ shield configurations.

$(\Rightarrow)$ Let $\left(\left(P^{1}, \tau^{1}\right),\left(P^{2}, \tau^{2}\right) \ldots\left(P^{T}, \tau^{T}\right)\right)$ be an optimal solution (that is such that diff $\left.=|V|^{2}\right)$. By Lemma 5, we know that $T \geq|V|$. Suppose w.l.o.g. that $T=|V|$.

In the following, for ease, we say that a step $t$ contributes to a sequence $V_{i}$ if the block $V_{i}$ is irradiated at step $t$ (the $i^{t h}$ bit of its mask $P^{t}$ is set to 1 ) and $t$ minimizes a sequence $V_{i}$ if the step $t$ is the last one of the treatment plan contributing to $V_{i}$ : at step $t-1$ and before, $V_{i}$ did not reach its minimum yet, at step $t+1$ and after, $V_{i}$ cannot be lowered. Note that a sequence is minimized at exactly one step. We first show that in one step we cannot minimize more than one sequence $V_{i}$, which is a corollary of the following proposition.

Proposition 2. If a sequence $V_{j}$ is minimized at step $t$, then this step cannot contribute to any $V_{i}, i<j$.

Proof. Let $V_{j}$ be the greatest sequence minimized at a step, $t$, also contributing to a $V_{i}, i<j$. Then $\tau^{t} \leq 1+q_{i}<q_{j}$, so other contributing steps to $V_{j}$ exist. As $V_{j}$ is the highest sequence sharing a dwell time with a lower sequence, the missing contributions can only be from steps minimizing lower sequences. Thus, the sum of these other contributions is bounded by $\sum_{j^{\prime}=1}^{j^{\prime}=j-1}\left(1+q_{j^{\prime}}\right)$. This leads to an upper bound for the sum of all contributions received by $V_{j}$ : $\sum_{j^{\prime}=1}^{j-1}\left(1+q_{j^{\prime}}\right)+\tau^{t} \leq \sum_{j^{\prime}=1}^{j-1}\left(1+q_{j^{\prime}}\right)+1+q_{i} \leq \sum_{j^{\prime}=1}^{j}\left(1+q_{j^{\prime}}\right)<q_{j}$. Then, the total irradiation time received by $V_{j}$ is not enough for it to be minimized at step $t$, which contradicts our starting hypothesis. 
Corollary 3. There exists no step minimizing more than one sequence.

Proof. According to Proposition 2, if two sequences $V_{i}$ and $V_{j}, i<j$ are minimized at the same step, then this step is both minimizing $V_{j}$ and contributing to $V_{i}$, which is impossible.

Since we have $|V|$ sequences and $|V|$ steps, exactly one $V_{i}$ is minimized at each step of the treatment plan. Without loss of generality, let us order the steps of our treatment plan such that each sequence $V_{i}$ is minimized at step $i$. In the next proposition, we prove that the dwell time at step $i$ can take one of two possible values, thus corresponding to a true/false assignment of variable $i$.

Proposition 3. For any $i, \tau^{i} \in\left\{q_{i}, 1+q_{i}\right\}$.

Proof. We prove the result by induction. From Proposition 2, it follows that $q_{i}-\sum_{j=1}^{i-1} \tau^{j} \leq \tau^{i} \leq 1+q_{i}$ for each step $i$. Thus, for the first induction step, it holds that $q_{1} \leq \tau^{1} \leq 1+q_{1}$, so $\tau^{1} \in\left\{q_{1}, 1+q_{1}\right\}$.

Considering the step $j$, and the sequence $V_{j-1, j}=\left(q_{j-1}+q_{j}, q_{j-1}+q_{j}+1\right)$, no step after $j$ can contribute to $V_{j-1, j}$ since

$$
\begin{aligned}
\tau^{j+1} \geq q_{j+1}-\sum_{i=1}^{j}\left(1+q_{i}\right) & \geq 1+2 \times \sum_{i=1}^{j}\left(1+q_{i}\right)-\sum_{i=1}^{j}\left(1+q_{i}\right) \\
& >q_{j-1}+q_{j}+2
\end{aligned}
$$

Moreover, $\sum_{i=1}^{j-1} \tau^{i} \leq \sum_{i=1}^{j-1}\left(1+q_{i}\right)<q_{j}$, so the contribution of step $j$ is mandatory to minimize $V_{j-1, j}$ which induces that

$$
\begin{aligned}
\tau^{j} \geq q_{j-1}+q_{j}-\sum_{i=1}^{j-1} \tau^{i} & \geq q_{j-1}+q_{j}-\sum_{i=1}^{j-1}\left(1+q_{i}\right) \\
& \geq q_{j}-1-\sum_{i=1}^{j-2}\left(1+q_{i}\right)
\end{aligned}
$$

Suppose now that there exists $k \geq 2$ such that $\tau^{j} \geq q_{j-1}-1-\sum_{i=1}^{j-k}\left(1+q_{i}\right)$. Consider then $V_{j-k, j}=\left(q_{j-k}+q_{j}, q_{j-k}+q_{j}+2\right)$. Applying a similar reasoning as before, we conclude that the contribution of step $j$ is mandatory, and, with our last lower bound over $\tau^{j}$ that

$$
\begin{aligned}
q_{j-k}+q_{j}-\tau^{j} & \leq q_{j-k}+q_{j}-\left(q_{j}-1-\sum_{i=1}^{j-k}\left(1+q_{i}\right)\right) \\
& \leq q_{j-k}+1+\sum_{i=1}^{j-k}\left(1+q_{i}\right)<q_{j-k+1}
\end{aligned}
$$


Thus, steps strictly between $j-k$ and $j$ cannot contribute. Therefore, the only steps able to contribute are $j$ and 1 to $j-k$ :

$$
\begin{aligned}
\tau^{j} \geq q_{j-k}+q_{j}-\sum_{i=1}^{j-k} \tau^{i} & \geq q_{j-k}+q_{j}-\sum_{i=1}^{j-k}\left(1+q_{i}\right) \\
& \geq q_{j}-1-\sum_{i=1}^{j-(k+1)}\left(1+q_{i}\right)
\end{aligned}
$$

We obtain a greater lower bound for $\tau^{j}$. This reasoning can be applied as long as $V_{j-k, j}$ exists, that is as long as $j-k \geq 1$. The last application $(k=j-1$, $k+1=j$ ) leads to

$$
\tau^{j} \geq q_{j}-1-\sum_{i=1}^{j-j}\left(1+q_{i}\right) \geq q_{j}-1
$$

On the whole, we obtain $\tau^{j} \in\left\{q_{j}-1, q_{j}, 1+q_{j}\right\}$. Moreover, if $\tau^{j}=q_{j}-1$, then step $j$ is not enough to minimize $V_{j}=\left(q_{j}, 1+q_{j}\right)$ (an amount of 1 or 2 is missing). But we can only use the dwell times of the treatment plan, and the lowest one is $\tau^{1} \in\{|V|,|V|+1\}$, where $|V|$ is the number of variables in $\phi$ (so $|V| \geq 3$ ). Thus, $\tau^{j}=q_{j}-1$ is impossible. This leads to $\tau^{j} \in\left\{q_{j}, 1+q_{j}\right\}$ for any step $j$.

To complete our proof, it remains to show that a sequence $C_{m}=\left(q_{a}+q_{b}+q_{c}+\right.$ $\left.2, q_{a}+q_{b}+q_{c}+2\right)$ cannot be minimized by other steps, except those corresponding to an assignment to the variables $a, b$ and $c$.

Proposition 4. Minimizing a sequence $C_{m}=\left(q_{a}+q_{b}+q_{c}+2, q_{a}+q_{b}+q_{c}+2\right)$ implies the contribution of exactly the steps $a, b$, and $c$.

Proof. W.l.o.g. let $a<b<c$. To minimize $C_{m}$, we need to apply a total amount of exactly $\tau=q_{a}+q_{b}+q_{c}+2$. Since $\tau^{c+1} \geq q_{c+1} \geq 1+2 \times \sum_{i=1}^{c}\left(1+q_{i}\right)>\tau$, step $c+1$ or higher cannot contribute to $C_{m}$. Thus the contribution of step $c$ is mandatory since $\sum_{i=1}^{c-1} \tau^{i} \leq \sum_{i=1}^{c-1}\left(1+q_{i}\right)<1+2 \times \sum_{i=1}^{c-1}\left(1+q_{i}\right)<q_{c}$. Similarly, $\tau^{b+1} \geq q_{b+1}>\tau-\tau^{c}$, so steps strictly between $b$ and $c$ cannot contribute, and $\sum_{i=1}^{b-1} \tau^{i}<q_{b} \leq \tau-\tau^{c}$ inducing that the contribution of step $b$ is mandatory. Finally, $\tau^{a+1} \geq q_{a+1}>\tau-\tau^{c}-\tau^{b}$, so steps strictly between $a$ and $b$ cannot contribute, implying that the contribution of step $a$ is mandatory since $\sum_{i=1}^{a-1} \tau^{i}<q_{a} \leq \tau-\tau^{c}-\tau^{b}$.

Gathering the previous results, we have an optimal solution to our MiNFIXMASKS instance if and only if each sequence $C_{m}$ corresponding to a clause $\left(v_{a}, v_{b}, v_{c}\right)$ receives exactly the dwell times received by the sequences $V_{a}, V_{b}$ and $V_{c}$. Moreover, each of theses $V_{i}$ receives either $q_{i}$ or $1+q_{i}$ as a (total) dwell time. Finally, minimizing $C_{m}$ implies that exactly one of the three $V_{i}$ receives the lowest of its two possible values. This corresponds to a truth assignment over $\phi$ such that each of its clauses contains exactly one true variable. 


\section{References}

1. T. Cormen, C. Leiserson, R. Rivest, and C. Stein. Introduction to Algorithms. MIT Press, 2014.

2. M. A. Ebert. Possibilities for intensity-modulated brachytherapy: technical limitations on the use of non-isotropic sources. Physics in Medicine and Biology, 47(14):2495, 2002.

3. Y. Liu, R. T. Flynn, Y. Kim, H. Dadkhah, S. K. Bhatia, J. M. Buatti, W. Xu, and X. Wu. Paddle-based rotating-shield brachytherapy. Medical Physics, 42(10), 2015.

4. Y. Liu, R. T. Flynn, Y. Kim, W. Yang, and X. Wu. Dynamic rotating-shield brachytherapy. Medical Physics, 40(12), 2013.

5. Y. Liu, R. T. Flynn, W. Yang, Y. Kim, S. K. Bhatia, W. Sun, and X. Wu. Rapid emission angle selection for rotating-shield brachytherapy. Medical Physics, 40(5), 2013.

6. R. Potter, C. Haie-Meder, E. V. Limbergen, I. Barillot, M. D. Brabandere, J. Dimopoulos, I. Dumas, B. Erickson, S. Lang, A. Nulens, P. Petrow, J. Rownd, and C. Kirisits. Recommendations from gynaecological (gyn) \{GEC $\{$ ESTRO $\}$ working group (ii): Concepts and terms in $3 \mathrm{~d}$ image-based treatment planning in cervix

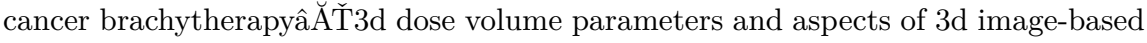
anatomy, radiation physics, radiobiology ", journal = "radiotherapy and oncology. 78(1):67 - 77, 2006.

7. T. J. Schaefer. The complexity of satisfiability problems. In Proceedings of the Tenth Annual ACM Symposium on Theory of Computing, STOC '78, pages 216-226, New York, NY, USA, 1978. ACM.

8. P. Smith, M. Klein, H. Hausen, and P. Lovoi. Radiation therapy apparatus with selective shielding capability, Jan. 2008. US Patent App. 11/471,277.

9. M. Webster, D. Scanderbeg, T. Watkins, J. Stenstrom, J. Lawson, and W. Song. Su-f-bra-11: Dynamic modulated brachytherapy (dmbt): Concept, design, and application. Medical Physics, 38(6):3702-3702, 2011.

10. M. J. Webster, S. Devic, T. Vuong, D. Yup Han, J. C. Park, D. Scanderbeg, J. Lawson, B. Song, W. Tyler Watkins, T. Pawlicki, and W. Y. Song. Dynamic modulated brachytherapy (dmbt) for rectal cancer. Medical Physics, 40(1), 2013.

11. M. J. Webster, D. J. Scanderbeg, W. T. Watkins, J. Stenstrom, J. D. Lawson, and W. Y. Song. Dynamic modulated brachytherapy (dmbt): Concept, design, and system development. Brachytherapy, 10, Supplement 1:S33 - S34, 2011. Abstracts of the 32nd Annual Meeting of the American Brachytherapy Society April 14-16, 2011.

12. W. Yang, Y. Kim, X. Wu, Q. Song, Y. Liu, S. K. Bhatia, W. Sun, and R. T. Flynn. Rotating-shield brachytherapy for cervical cancer. Physics in Medicine and Biology, 58(11):3931, 2013. 


\section{Appendix}

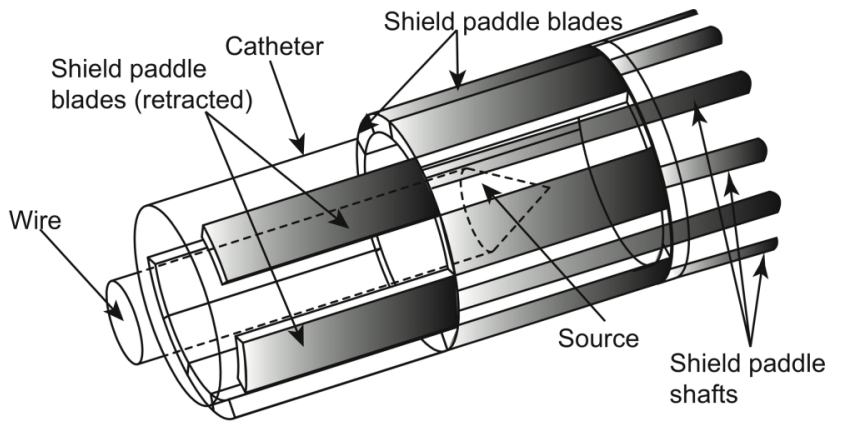

(a)

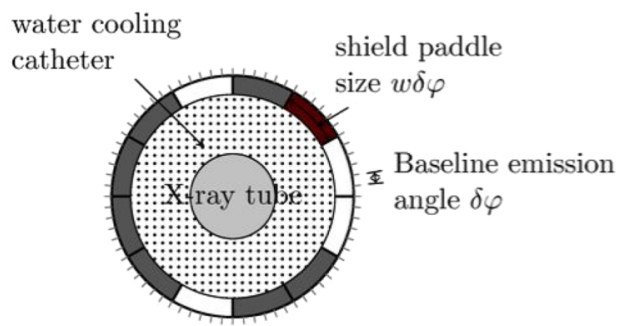

(b)

Figure 3: Figure 1 of [3] - A conceptual design of a P-RSBT applicator (a) 3D view. For the purpose of legibility, not all paddle shafts are drawn. (b) Cross-sectional view.

Lemma 1 For a sequence $S$ of natural numbers and a natural number $x$ consider the function $f(x)$ with $f(x)=\sum_{s \in S}|s-x|$. Then $f(x)$ has a unique minimum, which is only reached by any number $x$ in between the at most two medians of $S$. Moreover, for any $x$ not between the at most two medians of $S$, the function $f(x)$ decreases with the distance of $x$ to a median of $S$.

Proof. Let $m$ be a number inbetween the at most two medians of $S$ (if $S$ has only one median, then $m$ is equal to that median) and let $x$ be any natural number that is not inbetween the at most two medians of $S$. We denote by $S_{\leq x}$ the set of natural numbers $s$ in $S$ with $s \leq x$ and similarily with $S_{>x}$ the set of natural numbers $s$ in $S$ with $s>x$. We first show that $f(x)>f(m)$. We distinguish two cases: (1) $x$ is larger than the largest median of $S$ and (2) $x$ is smaller than the smallest median of $S$. In the first case $|s-x| \geq|s-m|+|x-m|$ for every $s \leq x$ 
and $|s-m| \leq|s-x|+|x-m|$ for every $s>x$. Moreover, because $x$ is larger than any median of $S$, we obtain that $\left|S_{\leq x}\right|>\left|S_{>x}\right|$. Hence

$$
\begin{aligned}
f(x) & =\sum_{s \in S}|s-x| \\
& \geq \sum_{s \in S \wedge s \leq x}|s-m|+|x-m|+\sum_{s \in S \wedge s>x}|s-m|-|x-m| \\
& =\sum_{s \in S}|s-m|+\left|S_{\leq x}\right||x-m|-\left|S_{>x}\right||x-m| \\
& >\sum_{s \in S}|s-m| \\
& =f(m)
\end{aligned}
$$

The proof for the second case is symmetric. This shows that a minimum of $f(x)$ can only be reached if $x$ is a number inbetween the two median of $S$ and that if $x$ is not inbetween the at most two medians of $S$, then $f(x)$ decreases with the distance of $x$ to a median of $S$. Hence in the case that $S$ has exactly one median there is nothing else to show. Now consider the case where $S$ has exactly two medians say $m_{1}$ and $m_{2}$. In this case $\left|S_{\leq m_{1}}\right|=\left|S_{\geq m_{2}}\right|$ and hence we obtain the following for any number $m$ with $m_{1} \leq m \leq m_{2}$.

$$
\begin{aligned}
f\left(m_{1}\right) & =\sum_{s \in S}\left|s-m_{1}\right| \\
& =\sum_{s \in S \wedge s \leq m_{1}}\left|s-m_{1}\right|+\sum_{s \in S \wedge s \geq m_{2}}\left|s-m_{1}\right| \\
& =\sum_{s \in S \wedge s \leq m_{1}}|s-m|+\left|m-m_{1}\right|+\sum_{s \in S \wedge s \geq m_{2}}|s-m|-\left|m-m_{1}\right| \\
& =\sum_{s \in S \wedge s \leq m_{1}}|s-m|+\left|S_{\leq m_{1}}\right|\left|m-m_{1}\right|-\left|S_{\geq m_{2}}\right|\left|m-m_{1}\right| \\
& =\sum_{s \in S}|s-m| \\
& =f(m)
\end{aligned}
$$

This shows that $f(x)$ has a unique minimum which is only reached by any number $x$ inbetween the at most two medians of $S$.

Lemma 3 Let $S$ be a set of dwell times. Then an $S$-restricted treatment plan minimizing $\sum_{n=0}^{N-1}\left|\hat{d}_{n}-d_{n}\right|$ can be found in time $O\left(\left(\hat{d}_{\max }\right)^{2}|S|+w^{2} \log w+\right.$ $\left.K \hat{d}_{\text {max }}\right)$. Moreover, an S-restricted treatment plan minimizing $\sum_{n=0}^{N-1}\left|\hat{d}_{n}-d_{n}\right|$ under the additional constraint $\hat{d}_{n}-d_{n} \geq 0$ for every $0 \leq n \leq N-1$ can be found in $O\left(\left(\hat{d}_{\max }\right)^{2}|S|+K w+K \hat{d}_{\max }\right)$ time. Moreover, if $\bar{S}$ is complete then the $S$-restricted treatment plans returned by the above algorithms are optimal among all (not necessarily $S$-restricted) treatment plans. 
Proof. Because every treatment plan has to apply the same dose to every patient volume under the same paddle, we obtain that $d_{i w}=d_{i w+1}=\cdots=d_{(i+1) w-1}$ for every $0 \leq i \leq K-1$. Hence, finding a treatment plan minimizing $\sum_{n=0}^{N-1}\left|\hat{d}_{n}-d_{n}\right|$ is equivalent to finding $K$ natural numbers $\overline{d_{0}}, \ldots, \overline{d_{K-1}}$ minimizing $\sum_{n=0}^{N-1}\left|\hat{d}_{n}-\overline{d_{\lfloor n / w\rfloor}}\right|$ such that each number $\overline{d_{i}}$ can be realized by a treatment plan. Since we are only considering $S$-restricted treatment plans, it holds that the set of realizable numbers (for any such treatment plan) is equal to the set of numbers that can be written as a sum of a subset of $S$. Moreover, because we can choose the masks arbitrarily it holds that for every $K$ numbers $\overline{d_{0}}, \ldots, \overline{d_{K-1}}$, which can be written as the sum of subsets of $S$, there is an $S$-restricted treatment plan realizing these numbers. To see this let $\overline{d_{0}}, \ldots, \overline{d_{K-1}}$ be any numbers such that for each $\overline{d_{i}}$ there is a subset $S\left(\overline{d_{i}}\right)$ of $S$ with $\overline{d_{i}}=\sum_{s \in S\left(\overline{d_{i}}\right)} s$, then $\left(\left(P^{1}, s_{1}\right), \ldots\left(P^{T}, s_{|S|}\right)\right)$ where $s_{1}, \ldots, s_{|S|}$ is an arbitrary ordering of the numbers in $S$ and the $i$-th bit of the binary string $P^{j}$ is 1 if and only if $s_{j} \in S\left(\overline{d_{i}}\right)$ is an $S$-restricted treatment plan realizing the numbers $\overline{d_{0}}, \ldots, \overline{d_{K-1}}$. It follows that given the set of allowed dwell times $S$, we can minimize each of the sums $\sum_{n=i w}^{(i+1) w-1}\left|\hat{d}_{n}-\overline{d_{\lfloor n / w\rfloor}}\right|$ separately for every $i$ with $0 \leq i \leq K-1$. Because of Lemma 1, the smaller $\sum_{n=i w}^{(i+1) w-1}\left|\hat{d}_{n}-\overline{d_{\lfloor n / w\rfloor}}\right|$ is, the closer $\overline{d_{\lfloor n / w\rfloor}}$ is to a median of the sequence $\hat{d}_{i w}, \ldots, \hat{d}_{(i+1) w-1}$. Moreover, if we consider the case where we have the additional constraint that $\hat{d}_{n}-d_{n} \geq 0$ for every $0 \leq n \leq N-1$, then the optimal value for $\overline{d_{\lfloor n / w\rfloor}}$ is the one that is closest to the minimum of the sequence $\hat{d}_{i w}, \ldots, \hat{d}_{(i+1) w-1}$. These considerations naturally lead to the following algorithm to find an $S$-restricted treatment plan minimizing $\sum_{n=0}^{N-1}\left|\hat{d}_{n}-d_{n}\right|$. The algorithm first computes a table that contains for every number $i$ between 1 and $\hat{d}_{\text {max }}$ either a subset $S^{\prime}$ of $S$ such that $i=\sum_{s \in S^{\prime}} s$ or Nil if no such subset $S^{\prime}$ exists for $i$. Using the standard text-book algorithm for the SUBSET Sum problem [1] (running in $O(s N)$ time, where $s$ is the sum that needs to be obtained and $N$ is the size of the set of integers), this can be achieved in $O\left(\left(\hat{d}_{\max }\right)^{2}|S|\right)$ time. In the case that we have no additional constraint the algorithm then computes the (at most two medians) $m_{i}^{1}$ and $m_{i}^{2}$ of the sequence $\hat{d}_{i w}, \ldots, \hat{d}_{(i+1) w-1}$ for every $0 \leq i \leq K-1$. This can be achieved (by sorting each of these sequences separately) in at most $O\left(w^{2} \log w\right)$ time. In the case that we have the additional constraint $\hat{d}_{n}-d_{n} \geq 0$ for every $0 \leq n \leq N-1$, the algorithm computes the minimum $\min _{i}$ for each of these sequences in $O(K w)$ time. Finally, the algorithm computes for every $0 \leq i \leq K-1$, the number $\overline{d_{i}}$ as the number between 1 and $\hat{d}_{\max }$ that has a non-nil entry in the table and either: (1) is closest to one of the medians $m_{i}^{1}$ and $m_{i}^{2}$ (in the case with no additional constraints) or (2) is closest to the minimum $\min _{i}$ (in the case that overdoses are not allowed). Using the table and the medians $m_{i}^{1}$ and $m_{i}^{2}$ respectively the minima $\min _{i}$ this can be achieved in time $O\left(K \hat{d}_{\max }\right)$. Considering an arbitrary ordering $s_{1}, \ldots, s_{|S|}$ of the numbers in $S$ and denoting by $S\left(\overline{d_{i}}\right)$ the subset of $S$ contained in the table for the number $\overline{d_{i}}$, the algorithm then outputs the treatment plan 
$\left(\left(P^{1}, s_{1}\right), \ldots\left(P^{T}, s_{|S|}\right)\right)$ where $s_{1}, \ldots, s_{|S|}$ is an arbitrary ordering of the numbers in $S$ and the $i$-th bit of the binary string $P^{j}$ is 1 if and only if $s_{j} \in S\left(\overline{d_{i}}\right)$. This completes the description of the algorithm. The running time of the algorithm is at most $O\left(\left(\hat{d}_{\max }\right)^{2}|S|+w^{2} \log w+K \hat{d}_{\max }\right.$ ) (in the case that overdoses are allowed) and $O\left(\left(\hat{d}_{\max }\right)^{2}|S|+K w+K \hat{d}_{\max }\right.$ ) (in the case that no overdoses are allowed). Finally, if $S$ is complete then the $S$-restricted treatment plan returned by the algorithm minimizes $\sum_{n=0}^{N-1}\left|\hat{d}_{n}-d_{n}\right|$ under all possible treatment plans. The same applies when considering the additional constraint $\hat{d}_{n}-d_{n} \geq 0$ for every $n$ with $0 \leq n \leq N-1$.

Lemma 5 To achieve a solution with diff $=|V|^{2}$ (which is the optimal solution one can get), one has to use at least $|V|$ different configurations.

Proof. The best solution in terms of irradiation differences is to apply to each $V_{i}$ a total dwell time $\tau_{i}$ corresponding to the median of $q_{i}$ and $1+q_{i}$, which is either $\tau_{i}=q_{i}$ or $\tau_{i}=1+q_{i}$, leading to a distance of 1 . Therefore, the minimal overall distance one can achieve for all the $V_{i}$ sequences is $|V|$.

Let us remark that, by construction, the $V_{i}^{\prime} s$ are increasingly ordered in $\hat{D}$ (i.e., $\left.V_{i}<V_{i+1}, 1 \leq i<|V|\right)$. Let $\left(\left(P^{1}, \tau^{1}\right),\left(P^{2}, \tau^{2}\right) \ldots\left(P^{T}, \tau^{T}\right)\right)$ be a treatment plan reaching the minimal overall distance $|V|$. Let us prove that $T \geq|V|$. Suppose that using $t<T$ different configurations of the treatment plan, one was able to apply a total dwell time $\tau_{i}$ corresponding to the median of $q_{i}$ and $1+q_{i}$ to each $V_{i}$ for $1 \leq i \leq j$. In other words, one has acheived the minimal overall distance for all $V_{i}$ for $1 \leq i \leq j$. Regarding $V_{j+1}$, the maximal overall contribution related to the use of these $t$ different configurations is lower or equal to $\sum_{i=1}^{j} 1+q_{i}$. Moreover, none of these $t$ configurations can be applied again since any of these contributes to at least one of $\left\{V_{1}, V_{2}, V_{j}\right\}$ and would increase the overall distance. Therefore, in order to reach the minimal distance for $V_{j+1}$, at least an extra configuration is needed. By induction, one cannot achieve the minimal overall distance of $|V|$ on the $V_{i}$ sequences without using at least $|V|$ different configurations.

Moreover, let us consider the $V_{*, i}$ sequences. Each $V_{i, j}=\left(q_{i}+q_{j}, q_{i}+q_{j}+2\right)$ has a minimum cost of 2 . Thus, the total cost of the $V_{*, i}$ sequences cannot be lower than $2 \cdot \frac{|V| \cdot(|V|-1)}{2}$. By adding the minimal cost for all the $V_{i}$ and $V_{*, i}$ sequences, one obtain an optimal diff $=|V|+|V| \cdot(|V|-1)=|V|^{2}$. 